\title{
PERFIL AFECTIVO-EMOCIONAL EN PROFESORES DE CIENCIAS ADMINISTRATIVAS HACIA CIENCIAS BÁSICAS
}

\section{AFFECTIVE-EMOTIONAL PROFLLE IN TEACHERS OF ADMINISTRATIVE SCIENCES TOWARDS BASIC SCIENCES.}

\author{
Henry de Jesús Gallardo-Pérez ${ }^{1}$ \\ Mawency Vergel Ortega ${ }^{2}$ \\ Carlos Sebastián Gómez Vergel ${ }^{3}$ \\ UFJC. Universidad de Los Andes
}

\section{RESUMEN}

La investigación sigue un enfoque cuantitativo, explicativo, correlacional, cuasi-experimental, transversal y de análisis multinivel que analiza la variabilidad entre los profesores de ciencias empresariales que trabajan en instituciones públicas y los que trabajan en instituciones privadas (nivel 1) y entre los dos tipos de instituciones (nivel 2), cuya hipótesis de partida establece que los profesores de ciencias empresariales de cada tipo de institución tienen competencias y emociones similares respecto a los que pertenecen al otro tipo de institución

1 Doctor en Educación, henrygallardo@ufps.edu. co, Orcid: 0000-0002-8239-1345, Docente Investigador. Universidad Francisco de Paula Santander. Cúcuta - Colombia. Grupo de investigación Arquimedes

2 Posdoctora en Investigación en Ciencias Sociales, Niñez y Juventud, mawency@ufps.edu.co Orcid: 0000-0001- 8285-2968, Docente-Investigadora. Universidad Francisco de Paula Santander, Cúcuta - Colombia. Grupo de investigación Graunt, Bioma

3 Ingeniería Electrónica (Estudiante). Universidad de Los Andes-Colombia. cs.gomezv@unian-des.edu. co orcid: https://orcid.org/0000-0002-6176-3613. Grupo de investigación Quetelet educativa. El objetivo de la investigación es identificar las emociones en los profesores de ciencias empresariales y sus factores determinantes. La muestra consistió en 90 profesores de ciencias empresariales, aplicando instrumento escala afectivo con 10 items, un instrumento estructurado con 20 ítems en escala nominal para medir las variables personales y las emociones y 20 ítems en escala Likert para analizar las competencias. Se encontró que existen diferencias significativas entre el personal según las instituciones con respecto a perfiles, competencias y las emociones, ya que el coeficiente de correlación intraclase del modelo nulo impide el cumplimiento de la hipótesis de independencia. En consecuencia, los profesores de ciencias empresariales del mismo tipo de institución tienen competencias y manejo de emociones similares en comparación con los de otra institución educativa; se incorporaron variables predictoras de los niveles: institución educativa y factores académicos 
del profesor de ciencias empresariales y su interrelación. La investigación concluye que existen tres perfiles de profesores que orientan en programas de ciencias empresariales, perfil asociado a la formación profesional y a la percepción hacia las ciencias básicas, así mismo, el modelo multinivel de emociones es adecuado para describir la relación entre las variables predictoras, las actividades orientadas a la enseñanza y el fomento de ambientes de aprendizaje que desarrollen competencias en emprendimiento y gerencia de empresa en los estudiantes de educación superior para promover el aprendizaje significativo y fortalecer la estrategia institucional basada en el manejo de las emociones de los profesores de ciencias empresariales.

\section{PALABRAS CLAVE}

Emociones, profesores, Factores, Modelos Multinivel, perfil

\section{$286 \quad$ ABSTRACT}

The research follows a quantitative, explanatory, correlational, quasi-experimental, crosssectional and multilevel analysis approach that analyzes the variability between business science teachers working in public institutions and those working in private institutions (level 1) and between the two types of institutions (level 2), whose starting hypothesis establishes that business science teachers in each type of institution have similar competencies and emotions with respect to those belonging to the other type of educational institution. The objective of the research is to identify emotions in business science teachers and their determinants. The sample consisted of 90 business science teachers, applying a structured instrument with 20 items on a nominal scale to measure personal variables and emotions and 20 items on a Likert scale to analyze competencies. It was found that there are significant differences between the personnel according to the institutions with respect to competencies and emotions, since the intraclass correlation coefficient of the null model prevents the fulfillment of the hypothesis of independence. Consequently, business science teachers from the same type of institution have similar competencies and emotion management compared to those from another educational institution; predictor variables of the levels were incorporated: educational institution and academic factors of the business science teacher and their interrelation. The research concludes that there are three profiles of professors who provide guidance in business science programs, a profile associated with professional training and the perception of basic sciences. The research estimates a multilevel model of emotions adequate to describe the relationship between the predictor variables, teaching-oriented activities and the promotion of learning environments that develop competencies in entrepreneurship and business management in higher education students to promote meaningful learning and strengthen the institutional strategy based on the management of emotions of business science teachers.

\section{KEYWORDS}

Emotions, Teachers, Factors, Multilevel Models

\section{INTRODUCCIÓN}

Existen muchos enfoques y orientaciones teóricas sobre la emoción; la mayoría de ellos consideran que las emociones son multifacéticas e implican la consideración de factores fisiológicos, cognitivos, sociales y conductuales [1]. La emoción es una reacción psicológica adaptativa, cuya finalidad es poner en marcha procesos psicológicos ante situaciones que exigen una respuesta rápida y eficaz para ajustarse a los cambios que se producen en el entorno [2]; puede entenderse desde varios puntos de vista, entre ellos, es posible entender una emoción como un proceso que se desencadena por la percepción de condiciones 
internas y externas con el fin de poner en marcha los recursos de los que se dispone para controlar una situación [3], también como un estado afectivo que el individuo experimenta acompañado de cambios orgánicos (fisiológicos y endocrinos) de origen innato o influenciado por la experiencia [4]. Los seres humanos pueden sentir emociones tanto positivas como negativas asociadas a la necesidad de actuar de una manera determinada. Las emociones tienen funciones adaptativas, sociales y motivacionales que confieren utilidad y permiten al sujeto ejecutar eficazmente reacciones conductuales adecuadas en la adaptación social y el ajuste personal [5]. Revisando la literatura, no se encuentra una clasificación única de las emociones básicas, la mayoría dice que son seis: alegría, ira, tristeza, asco, miedo y sorpresa [6]. El profesor de ciencias empresariales, como todo ser humano, experimenta diferentes emociones, sin embargo, a diferencia de los profesores de otras disciplinas, su interrelación con los alumnos es algo diferente ya que no sólo genera ambientes de aprendizaje de conocimientos específicos, sino que tanto él como sus alumnos interactúan en un ambiente en el que esto se vive en todo momento.

En este sentido, el estudio de las competencias se aborda también desde el conocimiento que el docente maneja desde su práctica pedagógica, el de la disciplina, cuya característica fundamental es la reflexión que realiza sobre el conocimiento producido en su campo disciplinar; el conocimiento pedagógico a través del cual comunica las reflexiones sobre la disciplina, y el conocimiento académico, caracterizado por el ejercicio personalizado resultante de los dos anteriores. Estos tres tipos de conocimiento se configuran por la práctica, el tiempo y la experiencia escolar y vital. Así, el conocimiento disciplinar, que responde a la pregunta "¿Qué sé?", el conocimiento pedagógico, ¿cómo enseño lo que sé? y el conocimiento académico, ¿Cómo me transformo con lo que sé? [7-9]
El propósito de la investigación, además de validar las escalas de evaluación de las emociones y las competencias, se dirige a establecer las relaciones existentes entre éstas y otras variables para establecer los factores asociados en la gestión de las emociones de los profesores de ciencias empresariales en la educación superior.

\section{MATERIALES Y MÉTODOS}

La investigación se enmarca en el paradigma cuantitativo y se incluye en un nivel explicativo, de campo, cuasi-experimental y transversal [10]. Se consideró como población de estudio a los profesionales que se desempeñan como docentes de ciencias empresariales en las diferentes instituciones educativas del departamento de Norte de Santander. La muestra estuvo conformada por 90 profesionales seleccionados de manera aleatoria estratificada y que manifestaron su consentimiento para diligenciar los instrumentos. Para la recolección de los datos se diseñó un cuestionario estructurado en dos partes: la primera dirigida a identificar los aspectos personales y emocionales de los profesionales de la enseñanza de ciencias empresariales, conteniendo 20 preguntas cerradas con dos o más opciones de respuesta mutuamente excluyentes. Un segundo instrumento correspondió a la identificación de competencias, para lo cual se basó en la formulación de 20 ítems en una escala Likert con cinco opciones de respuesta. Se ajusta a un modelo que identifica dos niveles de análisis: un primer nivel considera el conjunto de variables personales y competencias que afectan las emociones de cada docente; un segundo nivel contempla dimensiones referidas a competencias y emociones, cuyo efecto se expresa en conjunto sobre el docente en la institución en la que labora. El instrumento del cuestionario tiene una fiabilidad alfa de Cronbach $\alpha=0,8$, con fiabilidad para las competencias comportamiento individual y colectivo en 
relación con su práctica pedagógica $(\alpha=0,89)$, conocimiento disciplinar $(\alpha=0,91)$; conocimiento pedagógico $(\alpha=0,87)$; conocimiento académico $(\alpha=0,83)$; instrumento validado por el juicio de tres expertos que evaluaron la pertinencia, coherencia y redacción de los ítems, con índice kappa $\mathrm{K}=0,89$. Se realizó un análisis inferencial para validar la correcta selección de las variables y analizar la correlación o grado de asociación de las variables predictoras 0 independientes para determinar cuáles eran estadísticamente significativas para ser introducidas en la construcción del modelo estimado en dos niveles.

Las variables predictoras del nivel profesional docente fueron el género, la edad, la formación y la institución. Las variables de segundo nivel corresponden a las competencias derivadas del conocimiento disciplinar, el conocimiento pedagógico y el conocimiento académico. Se seleccionó como variable dependiente el manejo de las emociones (E), cuyo valor se obtuvo de la suma de los puntajes obtenidos en el cuestionario aplicado a los profesionales en cada una de las dimensiones (Xi) definidas por las emociones básicas, $. E=\sum_{i=1}^{6} X i$.

Se formuló el modelo ANOVA de efectos aleatorios para explicar a nivel de profesores de ciencias empresariales y a nivel de institución y para evaluar la bondad de ajuste de los modelos condicionales más complejos, se ajusta el modelo correspondiente al nivel 2 para conocer en qué medida las variables predictoras continuas explican las emociones de los profesores de ciencias empresariales. A continuación, se ajustó el modelo del nivel 1 para observar en qué medida las variables del profesorado predicen la variable dependiente [11] . Por último, se procedió a estudiar la interacción de ambos niveles para estimar el grado de interacción entre las variables a nivel de institución y las variables a nivel de profesor, comprobando los supuestos de normalidad y homogeneidad de varianzas, la evaluación de los estadísticos de influencia, el modelo y las estimaciones de los parámetros aleatorios [12]. Para estimar los parámetros desconocidos, se utilizó el procedimiento de Máxima Verosimilitud Restringida para obtener estimaciones insesgadas de los parámetros aleatorios, ya que el procedimiento de máxima verosimilitud, al no tener en cuenta la variación muestral de los parámetros fijos[13], produce estimaciones sesgadas de los parámetros aleatorios, lo que puede ser importante cuando el tamaño de la muestra es pequeño [14]. En cuanto a la significación de los componentes de la varianza, se utilizó la prueba $Z$ de Wald, donde la hipótesis nula afirma que las varianzas del modelo no son significativas frente a la alternativa de que las varianzas son significativas, y para los efectos fijos se utilizó la prueba t [15].

\section{RESULTADOS Y DISCUSIÓN}

Los valores de los coeficientes de correlación de Pearson $\left(\rho_{x, y}\right)$ entre las variables medidas en el nivel 1 de la jerarquía, es decir, los profesores de ciencias empresariales, para todas las variables predictoras muestran una correlación positiva alta y muy alta con la variable dependiente. Asimismo, se observa que no existe una correlación estadísticamente significativa [15] entre las variables predictoras. Por otro lado, en una segunda fase, se transformó cada una de las variables correspondientes a la valoración de las emociones para obtener una variable discreta dicotómica, tomando como punto de referencia el valor de la mediana, con un valor alto para los valores más altos y un valor bajo para los restantes. Se estableció la correlación biserial puntual $\left(r_{b p}\right)$ entre las variables dicotómicas del nivel 1 con estas nuevas variables independientes seleccionadas, encontrando una correlación significativa con la variable dependiente pero no significativa entre ellas. 
Perfil 1: Este grupo de profesores de áreas diferentes a ciencias empresariales, se caracteriza por tener un dominio afectivo hacia orientar módulos $u$ asignaturas en programas de ciencias administrativas en un grado medio, actitudes afectivo-emocionales, en un grado medio-bajo. La percepción que tienen de las ciencias básicas es nivel muy bajo, de alta dificultad, y manifiestan ansiedad alta producida al enfrentarse a cualquier situación problémica asociada a demostraciones. Emocionalmente podrían disfrutar con aplicación de ciencias básicas en ciencias administrativas pero acorde a la formación y campo de acción no les permite sentirse preparados para orientar cursos asociados a ciencias básicas aplicadas.

Perfil 2. Profesores con formación en ciencias administrativas, actitudes afectivo-emocionales, en un grado medio-alto; resultados altos en lo que se refiere al dominio afectivo-emocional hacia módulos y asignaturas en ciencias administrativas y nivel medio hacia orientar ciencias básicas específicamente matemáticas, manifiestan gusto medio por las ciencias básicas, un alto autoconcepto y manifiestan capacidad para resolver situaciones problémicas asociadas a las ciencias en aplicadas a administración y economía.
Perfil 3. Profesores con formación en ciencias administrativas, ingeniería y ciencias básicas que orientan módulos o asignaturas en programas de ciencias empresariales, actitudes afectivoemocionales, en un grado alto, con percepción alta hacia orientación módulos en ciencias administrativas y ciencias básicas aplicadas, algunos con formación postgradual en ciencias aplicadas a ciencias empresariales como tales como educación matemática, economía, estadística, actuaría, bioeconomía.

Para el análisis multinivel, según Quené y Van Den Bergh [13] del modelo nulo o de media incondicional, la gestión de las emociones de un profesor de ciencias empresariales se describe sin utilizar variables explicativas. Así, el modelo para cada nivel de emoción adopta una forma que depende de las variables explicativas [14]. A continuación, se estima el nivel de emociones de cada profesor y se realizan comparaciones para establecer el efecto que cada variable explicativa puede tener sobre estas emociones.

La figura 1 muestra el diagrama de barras de error que permite identificar la variación en los niveles de las emociones para los profesores de ciencias empresariales. Se puede observar que la variabilidad es similar en todos los tipos de emociones, pero con respecto al nivel, es mayor para las emociones alegría y tristeza, lo que indica que los profesores de ciencias empresariales no se preocupan mucho por la gestión de estas emociones, las dejan fluir. 


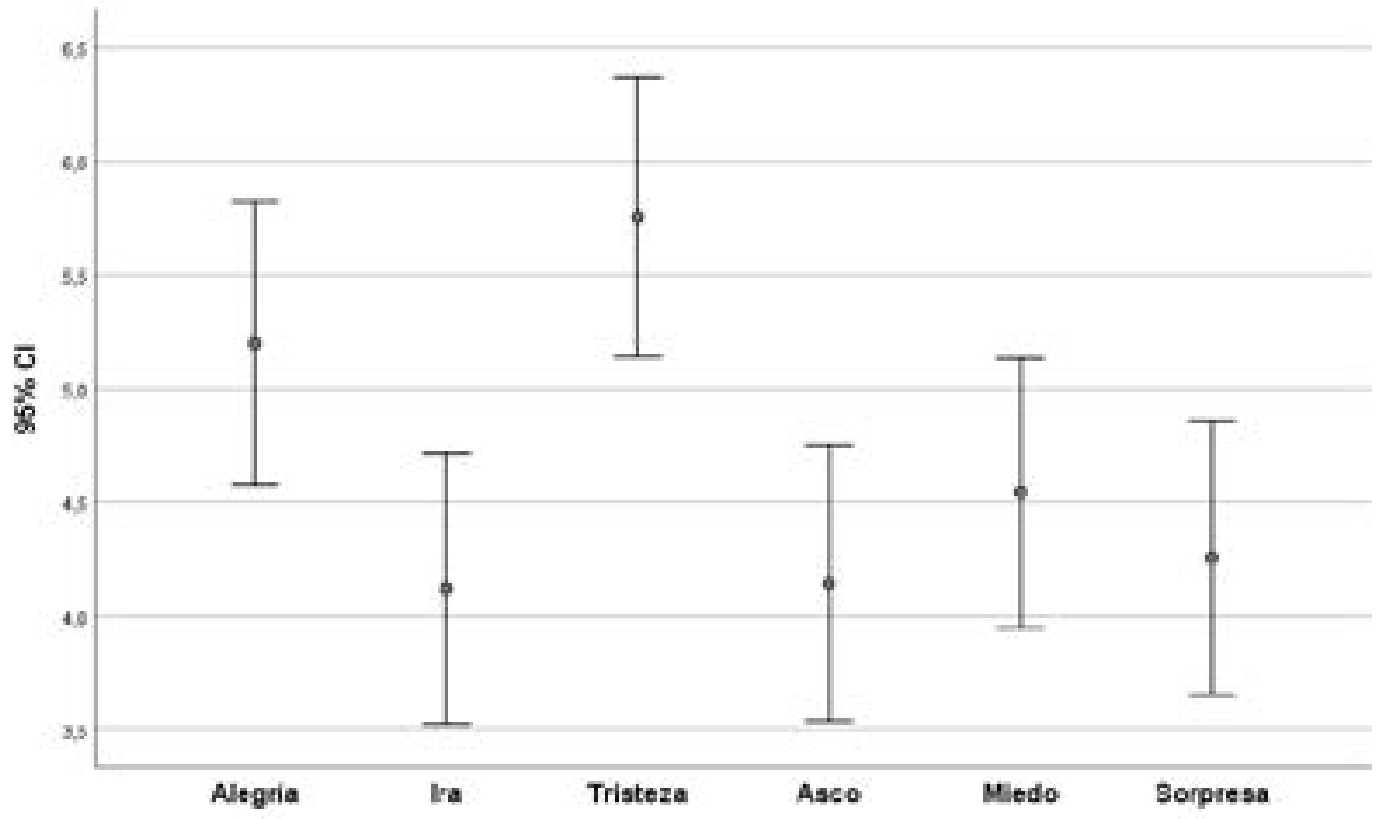

Figura 1. Estimación del nivel de emociones de los profesores de ciencias empresariales

La figura 2 muestra la variabilidad de las emociones para los profesores de ciencias empresariales según el tipo de institución, pública o privada. Se encuentran diferencias significativas en los niveles de las emociones ira y asco, lo que indica que, para estas emociones, la evaluación depende del tipo de institución, mientras que no hay diferencias significativas en los niveles de las demás emociones cuando se desagregan por tipo de institución.

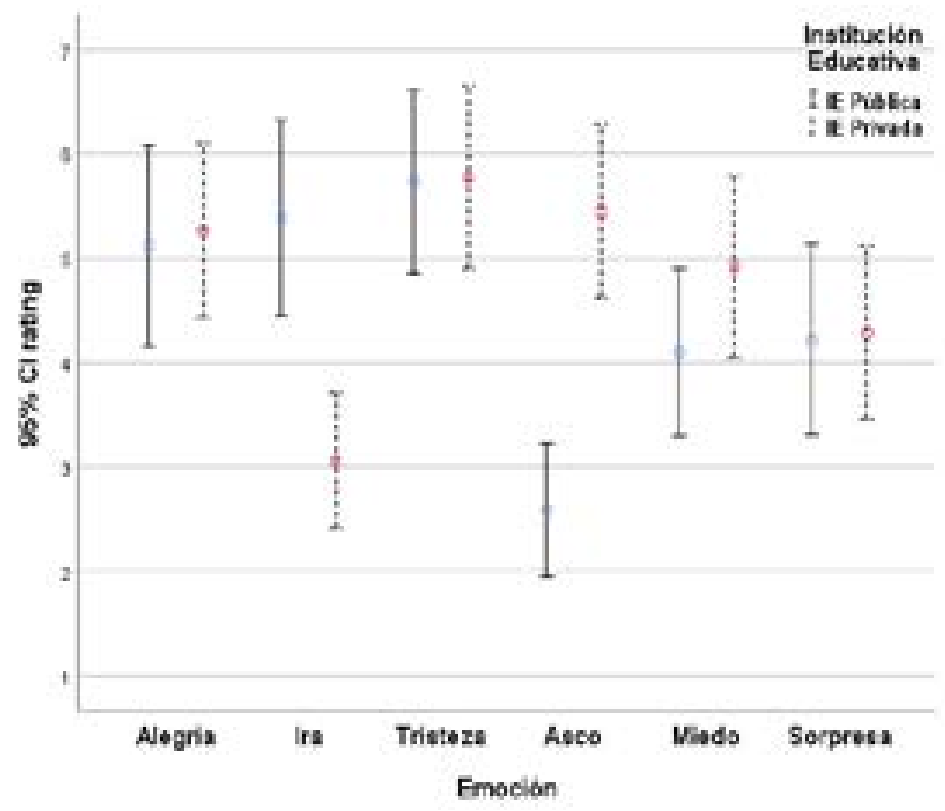

Figura 2 Nivel de emociones según el tipo de institución 
Un caso similar ocurre al diferenciar por los tipos de conocimiento que generan las competencias. Para cada tipo de conocimiento se identificaron tres niveles: bajo, medio y alto, con cortes en los percentiles 50 y 70 , respectivamente. Se encuentra, con un valor $p$ inferior al $5 \%$, que no hay diferencias en el manejo de las emociones para los profesores desagregados según su nivel de conocimiento disciplinar, excepto para la tristeza, que aparece con valores altos para los profesores con un nivel bajo de conocimiento disciplinar. En cuanto al conocimiento pedagógico, se encuentran diferencias en los niveles de valoración de las emociones tristeza y sorpresa. Para el conocimiento académico, las diferencias se encuentran en las valoraciones de las emociones tristeza y sorpresa.

Por último, la Figura 3 muestra un diagrama de correspondencias múltiples, que permite proyectar la nube de puntos multidimensional en un espacio bidimensional. Se establecen dos grupos con las modalidades de las variables analizadas que permiten diferenciar claramente la información correspondiente al tipo de institución educativa, pública y privada. En las instituciones públicas hay profesores que se caracterizan por tener altos niveles de valoración en las emociones asco y tristeza y bajos niveles en las emociones ira y alegría. En las instituciones privadas hay profesores con bajos niveles de valoración en las emociones asco, alegría y sorpresa y altos niveles de valoración en las emociones ira y tristeza. Las modalidades correspondientes a las variables género, conocimiento disciplinar, conocimiento pedagógico y conocimiento académico se sitúan en la parte central del gráfico, muy cerca del eje horizontal, por lo que no muestran diferencias entre los profesores que trabajan en los dos tipos de instituciones analizadas

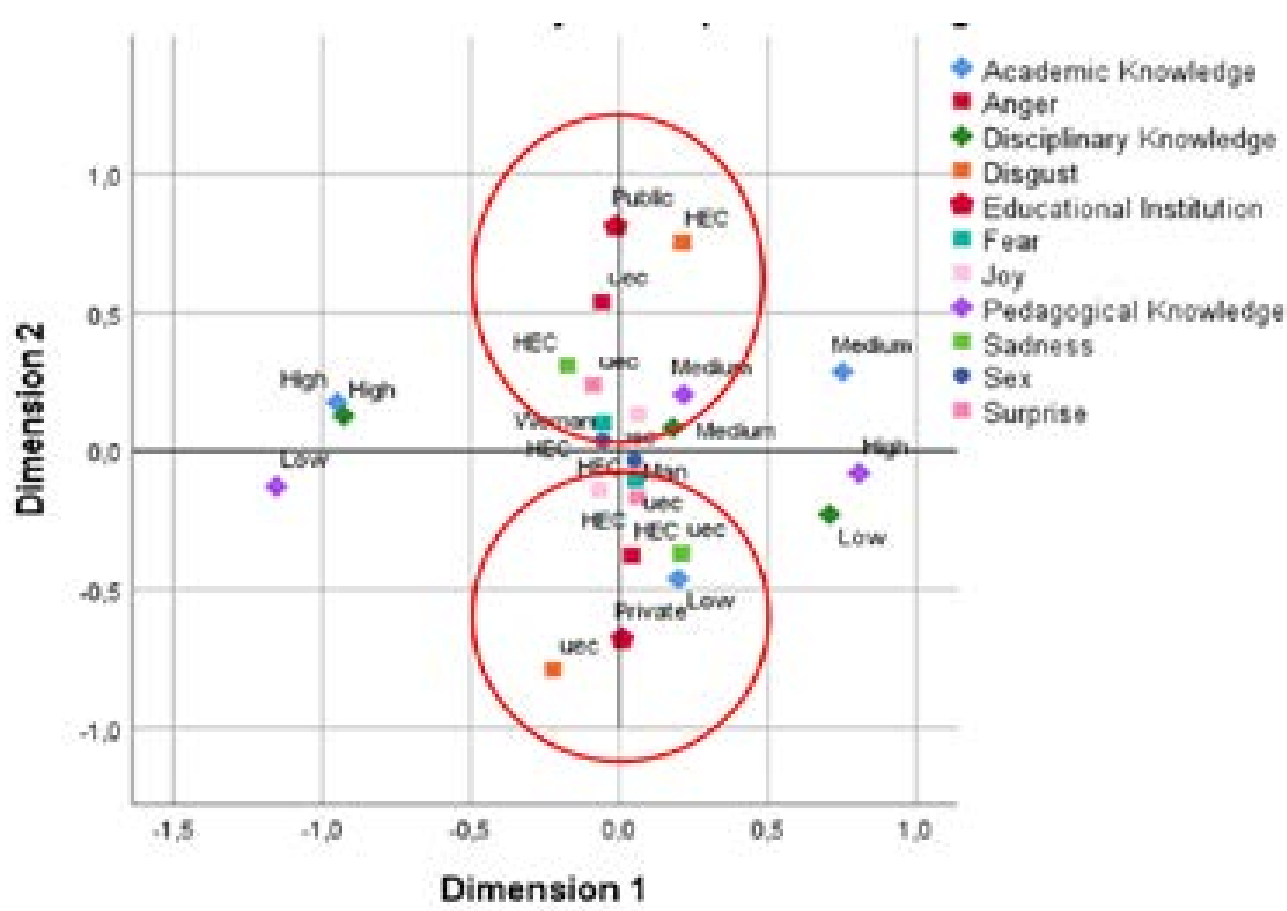

Figura 3 Correlaciones múltiples de los factores que influyen en las emociones 


\section{CONCLUSIONES}

Los resultados de la investigación permiten establecer que se cumple la hipótesis de que las emociones básicas se controlan de forma diferente entre los profesores que trabajan en instituciones públicas que entre los que trabajan en instituciones privadas. Se constata que, en el análisis de las emociones básicas, los profesores de ciencias empresariales que trabajan en instituciones públicas muestran poco interés por controlar las emociones de asco y tristeza, mientras que sí muestran control sobre la ira y la alegría. Los profesores que trabajan en instituciones privadas controlan poco las emociones de ira y tristeza y se preocupan más por controlar el asco, la alegría y la sorpresa.

Tres perfiles definen profesores, quienes pertenecen a áreas diferentes a ciencias empresariales, se caracteriza por tener un dominio afectivo en un grado medio hacia ciencias empresariales $y$, actitudes afectivoemocionales, en un grado bajo hacia ciencias básicas asociada a dificultad y ansiedad ante demostraciones; un segundo perfil profesores con formación en ciencias administrativas, actitudes afectivo-emocionales, en un grado medio-alto, y, profesores con formación en ciencias administrativas, ingeniería y ciencias básicas y postgrados asociados a ciencia, con actitudes afectivo-emocionales, en un grado alto.

No se encuentran diferencias significativas en los niveles de conocimiento en conocimientos disciplinares, pedagógicos y académicos en factores asociados a ciencias empresariales, pero si en afecto hacia las ciencias básicas, lo que permite concluir que no hay diferencias apreciables en las competencias del profesorado y que éstas no influyen en los niveles de valoración de las emociones en ciencias empresariales.
Esta investigación da pie a continuar con una segunda fase en la que se pueden establecer relaciones basadas en emociones secundarias que llevan a que los sentimientos del individuo en cada situación se complementen según sus características.

Conflicto de intereses: Los autores

manifiestan que no hay conflicto de intereses

\section{REFERENCIAS. BIBLIOGRÁFICAS}

[1] A. Sroufe, Desarrollo Emocional. México: Oxford, 2000

[2] A. Cano, A. Sirgio y M. Díaz, Control, defensa y expresión de emociones: Relaciones con salud y enfermedad. en E. G. Fernández-Abascal \& F. Palmero (Eds.), Emociones y Salud, Madrid: Ariel, 1999

[3] Vergel-Ortega, M. ., Gómez-Vergel, C. S. ., \& Caravalho-Casanova, J. F. . (2020). Cuentos sobre las emociones de jóvenes universitarios durante el proceso de aprendizaje del cálculo en ingeniería. Respuestas, 25(1), 89-95.

[4] E. Fernández-Abascal, B. Rodríguez, M. Jiménez, M. Martín y F. Domínguez, Psicología de la Emoción, Madrid: Editorial Universitaria Ramón Areces, 2018.

[5] Vergel, M., Gallardo, H. \& Martínez, J. (2014). Factores asociados al rendimiento académico en estadística de estudiantes de administración pública. Bogotá: Colección Pedagogía Iberoamericana.

[6] C. Izard, Basic emotions, relations among emotions, and emotion-cognition relations Psychological Review, vol. 99, pp. 561-565, 1992 
[7] P. Perenoud, Construire des compétences, tout un programme!, Revue vie Pédagogique, vol. 112, pp. 16-20, 1999.

[8] Vergel Ortega, M., Gómez Vergel, C. S., \& Gallardo Pérez, H. D. J. (2021). Resiliencia en mujeres universitarias en Norte de Santander, Colombia. Revista Latinoamericana De Ciencias Sociales, Niñez Y Juventud, 19(3), 1-19. https:// doi.org/10.11600/rlcsnj.19.3.4590.

[9] A. Zambrano, Tres tipos de saber del profesor y competencias: una relación compleja, Educere, vol. 10, no. 33, 225232, 2006.

[10] H. Gallardo, M. Vergel y F. Villamizar, Investigación intervención y enfoque multimétodo en ciencias humanas y educación matemática, Logos Ciencia y Tecnología, vol. 9, no. 2, pp. 84-96, 2017.

[11] Vergel, M. \& Martínez, J. (2013). Inteligencias múltiples y estilos de aprendizaje, su relación con el rendimiento académico de estudiantes de estadística. Revista Eco Matemático, 5 (1), 2014. 74-86.

[12] H. Goldstein, Multilevel statistical mode/s, Londres: Institute of Education. Multilevel Models Project, 1999.

[13] H. Quené y H. Van Den Bergh, On multilevel modeling of data from repeated measured designs: a tutorial, Speech Communication, vol. 43, pp. 103-121, 2004.

[14] E. Bologna, Tendencias en el análisis estadístico, Evaluar, vol. 11, pp. 59-84, 2012."
[15] J. Nieto, J. Rojas and M. Vergel. Impacto de estrategia pedagógica basada en el aprendizaje creativo para estudiantes de ingeniería. Editorial Ibáñez, 2019 